\title{
Actual Causes and Free Will
}

\author{
Carolina Sartorio \\ University of Arizona \\ BIBLID [0873-626X (2017) 45; pp. 147-165] \\ DOI: $10.1515 /$ disp-2017-0002
}

\begin{abstract}
In this paper I reexamine the debate between two contrasting conceptions of free will: the classical model, which understands freedom in terms of alternative possibilities, and a more recent family of views that focus only on actual causes, and that were inspired by Frankfurt's famous attack on the principle of alternative possibilities. I offer a novel argument in support of the actual-causes model, one that bypasses the popular debate about Frankfurt-style cases.
\end{abstract}

\section{Keywords}

Causation, actual causes, free will, alternative possibilities, Frankfurt.

\section{Introduction}

The focus of this paper is the contrast between two models of metaphysical freedom. One of the models is the classical view, which takes freedom to require the ability to do otherwise, or the agent's access to alternative possibilities of action. This idea is commonly known as the 'principle of alternative possibilities' or PAP. The opposing model is the conception spearheaded by Harry Frankfurt's famous attack on PAP (Frankfurt 1969), and it takes freedom to be only a function of the actual sources or the actual causes of action. In what follows, I refer to the two models as 'the AP model' and 'the AC model,' where 'AP' stands for alternative possibilities and 'AC' for actual causes. ${ }^{1}$

I developed a version of the AC model in my book Causation and Free Will (Sartorio 2016a). Here I look back at the contrast between

\footnotetext{
${ }^{1}$ van Inwagen 1983 and Ginet 1990 are examples of the AP model; Frankfurt 1969 and Fischer and Ravizza 1998 are examples of the AC model.

Disputatio, Vol. IX, No. 45, October 2017 
the two models. First I explain what I think is the best way to understand the terms of the debate. I then offer an argument in favor of the AC model, building on some features of the account of freedom developed in that book. (Note: this is a compatibilist account of freedom, that is to say, it is an account according to which acting freely is compatible with the truth of determinism. Although there are incompatibilist views that are based on alternative possibilities and also incompatibilist views that are based just on actual causes, here I am mostly interested in the debate between the two models assuming a compatibilist background.)

My main goal is to articulate the motivation for the AC model and to emphasize some aspects of the view that in my opinion make it a plausible conception of freedom. The standard way of looking at the debate between the two models revolves around an assessment of Frankfurt's original argument against PAP: the argument based on 'Frankfurt-style cases.' Here I discuss other aspects of the debate that are typically overshadowed by that issue, and that I consider to be at least as important, if not more important. ${ }^{2}$

\section{Two models of freedom}

I see the debate between the two models as fueled by a clash of intuitions.

On the one hand, it is natural to understand freedom in terms of having a range of options from which to choose. In particular, having alternative possibilities of action, or being able to do otherwise, appears to be required to be blameworthy for things, since it seems intuitively plausible to believe that we cannot be blameworthy for what we do if it is the only thing that we could have done. How could we blame people for doing something, if they could not have avoided doing it? These thoughts motivate the AP model of freedom.

However, on the other hand, imagine trying to absolve yourself of responsibility by pointing to factors that do not at all explain why you acted. This seems inappropriate: arguably, if a factor is completely irrelevant to why you acted, it cannot be used (by you or

${ }^{2}$ For other attempts to sidestep the debate over the prospects of Frankfurtstyle cases in refuting PAP, see Frankfurt 2003 and McKenna 2008. 
anyone else) to excuse your behavior. ${ }^{3}$ This motivates the idea that the only facts that are relevant to whether you acted freely and are responsible are facts about the actual sources of your behavior; thus it supports the AC model of freedom.

The two models are usually regarded as competitors, and rightly so. But first we need to understand exactly why they are rival views of freedom. This is an issue that has not been examined in enough detail in the literature, and it is of course central to understanding why there is a debate in the first place. So this is the issue that I will take up first.

The way I see it, the fact that the two models are competitors follows from certain plausible assumptions about the constitution of causal histories. Consider a Frankfurt-style case: a case of the kind originally used by Frankfurt (1969) to argue against the AP model of freedom, and in support of the AC model. Imagine that a neuroscientist is secretly monitoring your brain activity and wants you to make a certain choice: if you do not make it on your own, he will intervene by manipulating your brain in a way that guarantees that you will still make the choice (say, via the remote activation of a chip that he installed in your brain after having drugged you one night). Frankfurt argued that, if the neuroscientist does not need to intervene because you make the choice completely on your own, then you make the choice freely and are responsible for it, despite the fact that, given the neuroscientist's presence, you could not have done otherwise (i.e. you lacked alternative possibilities). If your choice is free despite the lack of alternative possibilities, it is because freedom is just a matter of the actual sources of action, as the AC model states. Frankfurt's idea is that, given that the neuroscientist never intervened, the actual causal sources of your behavior are the same

\footnotetext{
${ }^{3}$ This is a point originally made by Frankfurt. He wrote: '... there may be circumstances that make it impossible for a person to avoid performing some action without those circumstances in any way bringing it about that he performs that action. It would surely be no good for the person to refer to circumstances of this sort in an effort to absolve himself of moral responsibility for performing the action in question. For those circumstances, by hypothesis, actually had nothing to do with his having done what he did. He would have done precisely the same thing, and he would have been led or made in precisely the same way to do it, even if they had not prevailed.' (Frankfurt 1969, section V.)
} 
as if the neuroscientist had not been present, and they are enough to make your choice free.

At this point, there are a variety of responses open to advocates of the AP model. They could argue, for example, that, despite appearances to the contrary, the agent in a Frankfurt-style case does not act freely. Or they could argue that, despite appearances to the contrary, the agent has alternative possibilities, in the relevant sense. What is noteworthy for our purposes is that most contemporary advocates of the AP model would agree with Frankfurt that the presence of the neuroscientist does not affect the actual causal history of the agent's behavior in a Frankfurt-style case; still, they would insist that freedom is not exclusively grounded in actual causes but requires access to alternative possibilities of action. But a different way to resist Frankfurt could have been to reject the idea that the causal history of the act is unaffected by the neuroscientist's presence, and to argue, instead, that the agent's lack of alternative possibilities is somehow reflected in that causal history. If the lack of alternative possibilities were reflected in the actual causal history of the act in Frankfurt-style cases, then those cases could not be used to try and tease the two models apart, and the two models would collapse, at least with respect to such scenarios.

Consider, for example, the view that Ayer put forth in his classical paper on free will (Ayer 1954). Ayer defended a compatibilist view of freedom according to which we can act freely in a deterministic world to the extent that our acts are not constrained but are the result of our own choices. He embraced the simple conditional account of the ability to do otherwise according to which we could have done otherwise to the extent that, had we chosen otherwise, we would have done otherwise (which is compatible with determinism). But he also embraced an account of causation according to which $\mathrm{C}$ causes $\mathrm{E}$ if and only if $\mathrm{E}$ would not have happened if $\mathrm{C}$ had not happened. This is a simple counterfactual view of causation.

Such a view of causation entails that the actual causes of a choice in a Frankfurt-style case are not the same as the causes of the choice in a scenario where the neuroscientist is absent. For imagine that I make the choice on the basis of certain reasons in the neuroscientist-free scenario; those reasons cause my choice in that scenario. According to the simple counterfactual view of causation, those same reasons 
do not cause my choice in the scenario where the neuroscientist is present. For the counterfactual, 'If the reasons had not been present, I would not have made that choice', is false in that scenario (since the neuroscientist would have forced me to make the same choice, even in the absence of those reasons). Thus, if this view of causation were true, Frankfurt's reasoning could not even get off the ground, because the claim that the neuroscientist's presence does not affect the actual causal history of the agent's act would be simply false.

Nowadays almost everybody agrees that the simple counterfactual view of causation is false. In fact, this is precisely because of the conceivability of scenarios with the same structure as Frankfurtstyle cases where causes bring about overdetermined effects, or effects that do not counterfactually depend on those causes. ${ }^{4}$ But it is important to see that the claim that the AP model and the AC model are competitors rests on the assumption that this simple view of causation is false (a highly plausible assumption about the nature of the causal relation).

So far I have explained what makes the two models rival conceptions of freedom. This is important in order to understand why there is a debate between the two models. Now, also, in order to have a clear understanding of the debate between the two models, it is important to see the two views for what they are. I turn to this next.

Arguably, there is an important structural difference between the AP model and the AC model. On the one hand, as I anticipated above, the AP model aims to identify a central but only necessary condition for freedom, one that is captured by the following principle:

(PAP) Agents act freely only if they have alternative possibilities of action.

This principle is of course consistent with the claim that other things besides alternative possibilities matter to freedom, and are required for agents to act freely. In particular, PAP is consistent with the claim that facts about actual causes matter too. This is important because otherwise the AP model would be much less intuitively plausible than it is. Of course facts about actual causes, such as facts about how a choice was actually made, are relevant to freedom! It obviously

${ }^{4}$ For further discussion of this point, see Sartorio 2016a, chapter 2. 
matters to whether a choice was made freely if the agent made it as a result of, for example, practical reasoning or manipulation of some sort. So, on a charitable interpretation of the AP model, a view of that kind is not committed to the claim that facts about actual causes do not matter; all it is committed to is the claim that facts about alternative possibilities matter too. (Recall that, as I just illustrated with the Frankfurt-style examples, facts about alternative possibilities are something over and above just facts about actual causes, at least under certain plausible assumptions about causation.)

In contrast, the AC model aims to identify a class of facts - facts about actual causal histories - that constitute sufficient conditions for freedom. (This in turn seems to rule out other conditions, such as the alternative-possibilities condition, as necessary conditions for freedom.) Thus, whereas the AP model claims that alternative possibilities are necessary for freedom, the AC model claims that facts about actual causes are sufficient. This structural difference between the two models will play a significant role in the discussion that follows, so I will return to it below.

Finally, it will be helpful to say a bit more about the AC model and the commitments that lie at its core. Again, I think this is a major gap in the literature in the sense that, although some highly sophisticated approaches to freedom were developed in this tradition (notably, Fischer and Ravizza 1998), there has not been a sufficiently careful study of the fundamentals: the basic claims made by a view of that kind, and the central motivations behind them. I discuss this in detail in Sartorio (2016a, chapter 1), so here I will just focus on some of the main points.

In my view, the defining thought of the AC model is the following claim:

Exclusiveness: Freedom is exclusively grounded in facts about actual causes (and their grounds).

That is to say, all that matters to whether an agent acted freely is the relevant facts about the actual causes of the act, and whichever other facts may ground those facts about the actual causes.

Note that this is not just to say that freedom is exclusively grounded in facts about what actually happens, or the actual facts. It is rather the stronger claim that the only facts that ground freedom are a 
specific class of actual facts, namely, the causally explanatory facts. For example, an actual fact in a Frankfurt-style case is the presence of the neuroscientist with certain intentions. Still, according to the AC model this actual fact is irrelevant to the agent's freedom, because it is not connected with the actual causal explanation of the agent's choice.

According to the Exclusiveness claim, then, the only facts that are relevant to our freedom are facts concerning the actual causes of our behavior. Now, an important qualification may have to be made here depending on what one thinks about the possibility of causation involving absences. Some theorists think that absences cannot be genuine causes and effects; instead, they think that absences can at most play some secondary explanatorily relevant role (for example, Dowe suggests that absences are 'quasi-causes,' where quasi-causation is understood in terms of possible causation, and others think that absences can be relevant to causal explanations of phenomena without themselves being causes). ${ }^{5}$ Proponents of the AC model need a way to incorporate absences into their view, either as causes or as quasi-causes or the like, for absences play a significant role in the type of agency that is relevant to an agent's free will and responsibility. As an example of this, agents are sometimes morally responsible for what they omit to do (their omissions or inactions), and they are sometimes morally responsible for the outcomes of those omissions. But, arguably, omissions are absences: they are absences of actions of certain kinds. So, if there were no causation involving absences, the freedom-grounding facts would have to be expanded to include facts about quasi-causes, or any other kind of responsibility-grounding facts that could play the relevant kind of role. (I discuss another important example of the role played by absences below, in section 3.) Here I will assume, for simplicity, that absences can enter in causal relations, and thus that they can be a part of causal histories alongside positive events, but it is important to see that there are alternative ways of developing the view if it turned out that this is not the case. claim:

The Exclusiveness principle, in turn, entails a supervenience

${ }^{5}$ See Dowe 2001 and Beebee 2004, among others. 
Supervenience: Freedom supervenes on actual causes.

This means that, if two acts have the same actual causes, they are equally free or unfree. (Or perhaps a bit more strongly: if two acts have the same relevant actual causes, they are equally free or unfree.) Equivalently: there cannot be a difference in freedom without a (relevant) difference in the actual causes. Exclusiveness entails Supervenience because, if freedom is exclusively a matter of actual causes, and if two acts have the same actual causes (or if they have actual causes that are the same in all the potentially relevant respects), it follows that one of the acts is free just in case the other one is.

Again, Frankfurt-style cases can be used to illustrate this idea. Recall that Frankfurt's insight was that, if the neuroscientist never intervenes, the actual causal history of the agent's behavior is the same as if the neuroscientist had been absent, and that was an important part of the motivation for thinking that the agent's behavior is free. It is free because it is free in the scenario where the neuroscientist is absent, and the two behaviors have the same actual causal history; therefore, it is also free in the scenario where the neuroscientist is present.

So the main idea is this: according to the AC model, freedom is exclusively grounded in facts concerning the actual causal chain resulting in the act. The only facts that are relevant to whether an agent acted freely are facts about actual causes, and their grounds. In particular, facts about mere possibilities are not relevant to freedom. As noted above, this view has a lot of intuitive appeal. In particular, it is worth mentioning that even proponents of the AP model have felt the intuitive appeal of the Exclusiveness and Supervenience thoughts. Here is a quote from van Inwagen, for example:

[I]t would seem to be undeniable that if there are two possible worlds in each of which the same agent performs the same act, and if the causal history of this act is the same in every detail in both worlds, then it cannot be that the agent is responsible for that act in one world and not responsible for it in the other. (van Inwagen 1983: 164)

Here van Inwagen seems to be granting that the Supervenience claim, in particular, is extremely intuitive. ${ }^{6}$

\footnotetext{
${ }^{6}$ Of course, he then goes on to offer an argument in defense of the AP model,
} 
A moral that I think we can extract from all of this is that the fate of the AC model does not just rely on the success of Frankfurt's argument against PAP. Far from it, the AC model is a natural and elegant picture of freedom, one that is motivated by independently plausible considerations. Frankfurt-style cases can be used to illustrate the central thesis, but the view is plausible regardless of whether those cases can be successfully used to disprove the rival AP model. ${ }^{7}$ In short, the AC model is a promising alternative to the classical AP model.

Plus, there are some good reasons to prefer the AC model to the AP model. I turn to this in the next section.

\section{Argument for the AC model}

Recall that there is a structural difference between the two models: basically, whereas the AC model states that only actual causes are relevant to freedom, the AP model states that having alternative possibilities is also required. Thus there is an important sense in which the AC model is simpler than the AP model. In general, whenever we are faced with two models, one of which is simpler than the other, we should ask whether there is any good reason to prefer the more complex model; if there is no such reason, it makes sense to stick with the simpler one. So let's ask: is there any good reason to think that freedom requires access to alternative possibilities, in addition to a certain kind of actual causal history?

Of course, in the limited scope of this paper I cannot possibly examine every potential reason. So what I will do is use a strategy that should be familiar to those engaged in this debate: I will look at the potential role played by particular examples. As I mentioned above, much of the debate between proponents of the two models has focused on Frankfurt-style examples and whether they

which can be reconstructed as an argument against the Supervenience principle. I discuss the intuitive appeal of the supervenience claim in more depth in Sartorio 2016a, chapter 1. There I draw a distinction between a supervenience claim about freedom, or the metaphysical component of responsibility, and a supervenience claim about responsibility itself. See also Sartorio 2011.

${ }^{7}$ For further discussion of this point, see Sartorio 2017. 
can successfully disprove the AP model in favor of the AC model. I have suggested that the AC model is independently plausible and thus its fate should not hinge on the success or failure of Frankfurtstyle cases. However, given the role that examples have played in this debate, it seems relevant and quite fitting to turn the debate around and ask whether there could be examples that work as the counterparts of Frankfurt-style cases - that is to say, as examples that could be used to show, not that the AP model is false and the AC model is true, but that the AC model is false and the AP model is true. If such examples could be produced, this would of course be a good reason to prefer the more complex AP model to the simpler AC model. So in what follows I look at the question whether there are any such examples. Again, I am interested in this question because, if it were to be answered in the affirmative, this would be an obvious reason to prefer the AP model, and not because this is the only reason that there could be to prefer the AP model. There could be others, but I cannot examine them here.

In order to give the AP model the best shot at success, I will focus on one main type of scenario that is commonly used to illustrate the plausibility of that model. Although at first sight this type of scenario looks very promising, I argue that on reflection it does not support the alternative possibilities requirement; in fact, I argue that it supports the AC model.

Consider a case of irresistible compulsion: a man who, through no fault of his own, has become extremely addicted to a drug, and who on a certain occasion takes that drug out of the desire to take it. Given the extreme addiction, the man does not take the drug freely. ${ }^{8}$ Call him the addict. Compare him with another man who takes the drug out of his desire to take it, but who is not addicted to the drug. Given that he is not addicted to the drug, he takes the drug freely. Call him the non-addict.

The addict and the non-addict differ with respect to whether they take the drug freely. The AP model would account for this difference in freedom in terms of the ability to do otherwise: whereas the addict could not have refrained from taking the drug, the non-addict

${ }^{8}$ The assumption that the man has become addicted to the drug through no fault of his own is to ensure that he is not indirectly responsible for taking the drug. 
could have; this grounds the difference in freedom. On the face of it, this seems to be a very natural explanation of the difference between the addict and the non-addict.

Now consider: how can the AC model account for the difference in freedom between the addict and the non-addict? According to the Exclusiveness and Supervenience claims, any difference in freedom must be grounded in a (relevant) difference in the actual causes. But, what could this difference be? In both cases the agent takes the drug out of his desire to take it. Thus the challenge is to explain how there is still a difference in the actual causes of the act, one that can ground the difference in freedom between the addict and the non-addict. Recall that I am assuming a compatibilist framework, so I am assuming that the non-addict's act of taking the drug is free even if it has deterministic causes that trace back to a time before he was born. So the difference we are looking for is a difference between two sets of potentially deterministic actual causes: the causes of the addict's act and the causes of the non-addict's act.

Could the difference simply be that in one case the desire to take the drug is much stronger than in the other? If it made sense to think about the difference between the addict and the non-addict in just this way, as the addict being driven by a considerably stronger desire, this would make for a relevant difference in the actual causes of the act. Unfortunately, things are not that simple. For, presumably, how free an agent is while acting on a desire is not just a function of the strength of the desire, but it also depends on something like the agent's vulnerability to desires of a certain strength. And, on the face of it, it is far from clear how factors of that kind (factors that have to do with an agent's vulnerability or invulnerability to a desire) ought to be reflected in the actual causal history of the act of taking the drug, instead of in the agent's behavior in a range of possible situations different from the actual.

Fischer and Ravizza, in particular, would suggest that we should understand the difference between the addict and the non-addict in terms of the reasons-responsiveness of the actual 'mechanism' leading to the act in each case: the addict's actual mechanism is not responsive to reasons, but the non-addict's is. Their appeal to mechanisms and their properties has been a highly debated topic in the recent literature on free will. Without getting too much into this 
aspect of the Fischer-Ravizza view, it is worth noting for our purposes here that for Fischer and Ravizza the reasons-responsiveness of a mechanism is a function of the existence of merely possible worlds or circumstances where the mechanism yields different results. On the face of it, this seems to be in tension with the Exclusiveness and Supervenience principles, according to which all that is relevant to our freedom are facts about the actual causes of our behavior. ${ }^{9}$

At least initially, then, it appears that the AP model provides a better and more natural account of the contrast between the addict and the non-addict than the AC model. However, in what follows I argue that this is just an appearance. In fact, I argue that the opposite is true: the account that I suggest the AC model can provide of these cases is a better account, all things considered. But it has to be done in accordance with the principles that capture the original motivation for the AC model: the Exclusiveness and Supervenience principles.

The main idea is this. Instead of trying to explain the difference between the addict and the non-addict just in terms of the properties of the desire itself, such as its strength (which is arguably a misguided strategy), or in terms of how the agent reacts to the desire in other possible scenarios (which is irrelevant to an agent's freedom, according to Exclusiveness and Supervenience principles), we should capture it in terms of other elements of the actual causal history, different from the desire itself, but that also plausibly explain why the agent makes the choice on that occasion.

I suggest that certain absences of reasons play this role. When an agent is sufficiently sensitive to reasons, I suggest, her behavior is the result of reasons and absences of reasons. For example, when I decided to go for a walk this morning, my going for a walk was the result of my wanting to get some fresh air, but it was also the result of the phone not ringing a second earlier, a neighbor not knocking on my door asking for my immediate help, etc. These absences are just as much a part of the explanation of my behavior as my desire to get some fresh air, assuming that I am the kind of person who is moved by those kinds of reasons.

Similarly, a non-addict's decision to take the drug is the result

${ }^{9}$ For further discussion of this point, see Sartorio 2016a, chapter 4. 
of his desire to take the drug, but also, I suggest, of the absence of the relevant kinds of reasons. Nothing comes up that requires his immediate attention; this explains why he takes the drug. Imagine, for example, that if some family member had knocked on the door of the non-addict's house asking for his help (call this reason 'R1'), he would have helped them instead of taking the drug. Had a close friend called him on his cell with an urgent request (call this reason 'R2'), he would have taken the call instead of taking the drug. Had the fire alarm gone off suddenly (call this reason 'R3'), he would have immediately left the building, leaving the drug behind. Etcetera. In that case, the absence of R1, R2, R3, etc. causally explains the non-addict's act of taking the drug. An extreme addict, by contrast, would have been moved to take the drug even in the presence of reasons of that kind. So the absence of R1, R2, R3, etc. is not causally explanatory in his case. ${ }^{10}$

This means that whenever an act is done freely, it has many causes, and more causes than one might have initially realized. In particular, it means that it has more causes than if it had not been done freely! A free agent is someone who, in acting, is causally responding to a number of reasons and absences of reasons that rationalize her behavior. Her sensitivity to those reasons and absences of reasons is what makes her act free.

On this view, absences play an essential role in grounding an agent's freedom. The role that on the AP model is played by alternative possibilities is played here by actually explanatory absences (of reasons). ${ }^{11}$ If those absences of reasons are actually explanatory, if

\footnotetext{
${ }^{10}$ I discuss this in more detail in Sartorio 2015 and in Sartorio 2016a, chapter 4. There is at least one other way in which absences could be relevant to the resistibility of a desire. Imagine that, when a non-extreme addict feels the urge to take the drug, there are certain strategies he can use to divert his attention away from the drug. Imagine that doing Sudoku puzzles usually helps. Then, if on a certain occasion he decides not to do Sudoku puzzles and succumb to the urge, his not using those strategies is part of the causal explanation of his taking the drug. In this case his responsibility for taking the drug seems to be inherited from his responsibility for the decision not to do Sudoku puzzles. Thanks to Josh Cangelosi for discussion of examples of this kind.

${ }^{11}$ As I mentioned above, in section 2, the simplest view is one that takes that explanatory role to be a straightforward causal role. But there are other ways of
} 
they are indeed part of the actual causal chain, then, arguably, actual causes are all we need to ground the agent's freedom; we don't need to appeal to the existence of alternative possibilities at all. Why did the non-addict act freely when he took the drug? The answer is not that he could have done otherwise, but, rather, just a fact about actual causes: the fact that, when he took the drug, he was actually responding to certain reasons to take the drug as well as to certain absences of reasons not to take the drug. The addict, by contrast, was not sufficiently responsive to the absence of reasons not to take the drug.

One may wonder whether absences are really needed, or whether there are other positive elements of causal histories that could play the required role. How about, for example, appealing to agents' dispositions? Arguably, the addict and the non-addict have different dispositions, for instance, the non-addict has the disposition to attend to his family if they ask for help, and the addict doesn't. Could one argue that this difference in dispositions by itself explains the difference in freedom between their acts? ${ }^{12}$

I think the answer is 'no.' Although there is such a difference in dispositions, the non-addict's disposition is not actually manifested when he decides to take the drug. Nothing came up that required his immediate attention: in fact, his family did not ask for help, he did not get a call from a neighbor, and the fire alarm did not go off. It follows that his disposition to respond to such emergencies is not part of the actual explanation of his act of taking the drug. Thus, whereas there is a difference in the dispositions of the addict and the non-addict, this is not a difference in the actual causal history of the acts..$^{13}$ But the Supervenience principle states that, if there is a

understanding it, if it turns out that absences are never causes.

${ }^{12}$ McKenna (2013) proposes an actual-sequence view of responsibility that is focused on the dispositions of agents to respond to reasons.

${ }^{13}$ It is common to understand dispositions in causal terms. For example, an object's fragility is something like the disposition that the object has to show a certain manifestation (breaking) when it undergoes a certain stimulus (being dropped or struck). And it is common to understand the relation between the object's having the disposition and the object's manifesting that disposition (or, at least, between the object's having the intrinsic property that is the 'causal basis' 
difference in freedom between two acts, there must be a difference in the causal history of those acts. So the difference in dispositions cannot play that kind of role; the role must be played, instead, by the absences of the relevant reasons.

Surely, the difference in dispositions between the addict and the non-addict still plays some role. I think the role is this. In the nonaddict's case, the existence of the disposition helps ground the fact that the absence of certain reasons is causally relevant to the agent's behavior. That is to say, the absence of those reasons (R1, R2, R3, etc.) is causally relevant to the non-addict's behavior because, or in virtue of the fact that, the non-addict has the disposition to respond to those reasons. If the non-addict had lacked those dispositions, the absence of those reasons would not have been causally relevant to what he does, as in the case of the addict. So the disposition plays a role without itself being part of the causal chain. The absences of reasons are part of the causal chain, but they are part of the causal chain in virtue of the existence of those dispositions.

Recall that when I introduced the Exclusiveness principle I said that we should not understand it as just the claim that facts about freedom are exclusively grounded in facts about the actual causal history, but as the claim that facts about freedom are exclusively grounded in facts about the actual causal history and whatever other facts may ground those facts. Grounds of grounds are also grounds (at least typically) ${ }^{14}$, so some facts may ground facts about freedom in this more indirect way, by grounding facts about the causal history. As we can see now, the facts concerning the dispositions of the addict and the non-addict work as indirect grounds of that kind. The absences of the relevant reasons play the more direct grounding role, since they are part of the actual causal chain.

for the disposition and the object's manifesting that disposition) as a causal relation, when the stimulus obtains. (See, e.g., Lewis 1997.) But the point is that in the case of the non-addict the relevant stimulus does not obtain; hence the disposition is not manifested, and thus it is not part of the actual causal history of events.

${ }^{14}$ The transitivity of grounding has been contested in recent years. However, even those who have argued that grounding is not universally transitive have regarded the counterexamples as exceptions that need to be accounted for by a theory of grounding and have looked for some replacement principle or resolution, given how natural it is to see grounding as a transitive relation. See, e.g., Schaffer 2012. 
I have suggested that there is a difference in the actual causal history of the behavior between the addict and the non-addict, one that has to do with the role played by the absences of the relevant reasons. I have argued that advocates of the $\mathrm{AC}$ model can use that difference to ground the difference in freedom that seems to exist between the two agents. But, importantly for our purposes here, I think that everybody should accept that there is such a difference in the causal history. ${ }^{15}$ For it is just commonsense. It only takes a bit of reflection to see that, when I go out for a walk, the causal explanation of my behavior includes more than just the most salient reasons - say, the desire to get some fresh air. The fact that there was no family emergency that required me to stay at home is also part of the explanation, and so are many other facts of a similar kind. By contrast, if my cat walked out the door this morning, the absence of a family emergency or some pressing moral reason to stay at home will typically not be part of the explanation of his behavior, since cats are typically not responsive to those kinds of reasons. ${ }^{16}$ By the same token, there plausibly is a similar difference between the addict and the non-addict: the addict is not being responsive to certain facts that the non-addict is being responsive to. Rejecting this flies in the face of commonsense.

Not just this, but, also, these seem to be highly relevant differences: they are differences that intuitively bear on the agents' freedom and responsibility. It is very plausible to believe, for example, that the addict's non-responsiveness to the absence of a family emergency is relevant to why he does not act freely on that occasion. And similarly for the non-addict, it is very plausible to believe that the non-addict's responsiveness to the absence of a family emergency is relevant to why he acts freely on that occasion. Again, this is a fact that everybody should acknowledge, not just advocates of the $\mathrm{AC}$ model of freedom.

\footnotetext{
${ }^{15}$ In this respect, the view has a considerable advantage over other views that appeal to more controversial forms of causation or agency, such as agent-causal accounts.

${ }^{16}$ On the other hand, of course, cats can be responsive to the absence of other kinds of events (such as the absence of the sound of a tuna can being opened) to which humans are typically not responsive.
} 
Recall, once more, that I am interpreting the AP model as consistent with the claim that facts about actual causes can be relevant to freedom. If facts about actual causes can be relevant to freedom, and if there is an intuitively relevant difference of that kind between the addict and the non-addict, it seems that even an advocate of the AP model should acknowledge that there is a plausible account of the difference between the addict and the non-addict in terms of just the actual causal histories. An account in terms of alternative possibilities is no longer needed; indeed, it now seems superfluous, given the difference in the actual causal history.

Thus, I conclude that examples like the addict and the non-addict do not support the AP model; on reflection, and despite initial appearances, they in fact support the AC model. I predict that other potential counterexamples would fail for similar reasons. Of course, I cannot show this in the limited space I have here, but the fact that we have been able to establish this in a paradigm case goes some way towards suggesting that the result generalizes. ${ }^{17}$ Again, the fact that there are no successful counterexamples to the AC model does not by itself show that the AC model is true or that the AP model is false. What it does suggest is that a main kind of strategy that one could think of using against the AC model, a strategy based on counterexamples, is a non-starter. ${ }^{18}$

\section{Conclusions}

In this paper I have offered a series of considerations in support of

\footnotetext{
${ }^{17}$ In other work (Sartorio 2016c) I have called potential counterexamples to the AC model - in particular, examples that support the AP model over the AC model-'PAP-style cases.' In that paper I discuss other types of cases that could work as potential counterexamples to the AC model (and in support of the AP model), and I argue that they fail.

${ }^{18}$ This might also motivate a new kind of attitude toward the debate about Frankfurt-style cases. Regardless of which view of freedom is actually true, a counterexample-based strategy may not be the right way to settle this issue. Perhaps the debate should be settled instead in terms of the views' general motivation, how well they capture our intuitive judgments about specific cases, and/ or the extent to which they display a number of theoretical virtues. For further discussion of this point, see Sartorio 2016b and 2016c.
} 
the AC model of freedom. In doing so, I have completely sidestepped the debate about Frankfurt-style cases. If we cannot show that the AP model fails by appeal to Frankfurt-style cases (an issue that I have not tried to settle one way or the other in this paper), at least we can help bring out the promise of an alternative model of freedom, one based just on actual causes, and we can try to develop the basic insights into an internally consistent and independently motivated account of freedom.

I have tried to make some progress towards that goal. First, I noted that the AC model has considerable intuitive plausibility, and I explained how the fundamental idea can be expressed in terms of two main principles, Exclusiveness and Supervenience. I drew attention to the fact that the AC model is simpler than its rival, the AP model, when the latter is understood in the most charitable way. Then I examined one main kind of strategy for trying to show that we should abandon the simpler model in favor of the more complex one - the existence of counterexamples - and I argued that such a strategy fails. In fact, I argued that it backfires, for some paradigm cases that one could think of using as counterexamples, far from being real counterexamples, end up lending further support to the $\mathrm{AC}$ model of freedom. ${ }^{19}$

Carolina Sartorio

Philosophy Department, University of Arizona Social Sciences 213

Tucson AZ 85721, USA sartorio@arizona.edu

\section{References}

Ayer, A. J. 1954. Freedom and necessity. In his Philosophical Essays. London: Macmillan.

Beebee, H. 2004. Causing and nothingness. In Causation and Counterfactuals, ed.

${ }^{19}$ I am very grateful to Pablo Rychter and Josep Corbí for organizing the II Blasco Disputatio workshop at the University of Valencia in September of 2016, where I presented an earlier version of this paper, and to Carlos Moya and the audience at that workshop for the lively discussion. Thanks also to Juan Comesaña, Peter Graham, Michael McKenna, and audiences at the Australian National University, the Collège de France, and the University of Barcelona. 
by J. Collins, N. Hall, and L. A. Paul. Cambridge, Mass: MIT Press.

Dowe, P. 2001. A counterfactual theory of prevention and 'causation' by omission. Australasian Journal of Philosophy 79: 216-26.

Fischer, J. and M. Ravizza. 1998. Responsibility and Control. Cambridge: Cambridge University Press.

Frankfurt, H. 1969. Alternate possibilities and moral responsibility. Journal of Philosophy 66(23): 829-39.

Frankfurt, H. 2003. Some thoughts concerning PAP. In Moral Responsibility and Alternate Possibilities: Essays on the Importance of Alternative Possibilities, ed. by D. Widerker and M. McKenna. Aldershot: Ashgate.

Ginet, C. 1990. On Action. Cambridge: Cambridge University Press.

Lewis, D. 1997. Finkish dispositions. The Philosophical Quarterly 47: 143-58.

McKenna, M. 2008. Frankfurt's argument against alternative possibilities: looking beyond the examples. Noûs 42: 770-93.

McKenna, M. 2013. Reasons-responsiveness, agents and mechanisms. In Oxford Studies in Agency and Responsibility, vol. 1, ed. by D. Shoemaker. Oxford: Oxford University Press.

Sartorio, C. 2011. Actuality and responsibility. Mind 120(480): 1071-97.

Sartorio, C. 2015. Sensitivity to reasons and actual sequences. In Oxford Studies in Agency and Responsibility, vol. 3, ed. by D. Shoemaker. Oxford: Oxford University Press.

Sartorio, C. 2016a. Causation and Free Will. Oxford: Oxford University Press.

Sartorio, C. 2016b. A partial defense of the actual-sequence model of freedom. Journal of Ethics 20(1): 107-20.

Sartorio, C. 2016c. PAP-style cases. Journal of Philosophy 113(11): 533-49.

Sartorio, C. 2017. Frankfurt-style examples. In Routledge Companion to Free Will, ed by M. Griffin, N. Levy, and K. Timpe. London: Routledge.

Schaffer, J. 2012. Grounding, transitivity, and contrastivity. In Grounding and Explanation, ed by F. Correia and B. Schnieder. Cambridge: Cambridge University Press.

van Inwagen, P. 1983. An Essay on Free Will. New York: Oxford University Press. 